\title{
Human Behaviour in Emergency Egress of Building Fires
}

\author{
MYONG-O YOON and SHINICHI SUGAHARA \\ The University of Tokyo \\ Tokyo, Japan
}

KOICHI KISHITANI

Nihon University

Tokyo, Japan

ABSTRACT

This study aims to develop a practical methodology within the parameter of experimental psychology to obtain broader information between the interaction of humans and building environment during fire condition, and thus to find out the causes of psychological stress or physiopsychological injury in fire. In part I of the study we developed a visual experience simulator on the assumption that visual perception dominates psychological interactions between humans and building environment. Through a series of experiments with this simulator, it became possible to put the complete response of an individual under observation. Data showed the causes that led to the occupants' confusion of recognizing direction or standing point. This proposal should be helpful in evaluating the safety of building elements in light of the ease of perception on the part of the occupants. Part II, the aim of the study was to investigate the physiopsychological impact from the presence of co gas. By applying the theoritical model of experimental psychology, the existance of physiopsychological impact in case of acute $C O$ poisoning was identified. The experimental method proposed here and its results should be helpful in improving contemporary test methods. The methods estimate gas toxicity through comparison analysis based on the immobilazation of CO gas poisoned animals.

\section{INTRODUCTION}

Fire engineering is faced with an increase of both the complexity of building configurations and amouni of floor space due to larger sized projects that include a program for various functions. This situation should lead to a higher concern about safety and emergency evacuation in case of fire as well as human behavior in case of sudden disaster. Meanwhile the actual Iimits of experimental methodology in the conditions of real life situations remains rather problematic. Therefore, these means should be studied throughly. Here stands our proposal for a new methodology. We propose a theoritical model which reveals some risk that have not been taken into account on till now. In conclusion from these experiments, our methodology tried to palliate the lack of information concerning human behaviour and phisiopsychological effects in fire situations. From these results we should be able to make suggestions concerning safety and emergency evacuation systems in order to avoid some of the existing problems. 
PART I : Behaviour Study Viewed from Perceptional Response in Simulated Visual Environment

This part of the experimental study aims to find practical element as a method both for building design and to estimate the established building in view of safety egress. But when studying human behaviour in relation to emergency situations where people acting in a produced emergency situation two basic sequential problems araise. First no matter how complete an experimental environment we have, there will exist obvious distinctions between the real and the reproduced. What makes this problem more difficult is that it is very complicated to analyoe the obtained data in terms of an interpretation of the differences of the real and the simulated situations. The second problem is of experimental techniques. In general cases, human behaviour is observed in whole scale experimental structures. Whole scale experiments are supposed to offer more reliable data for interpretation, but the cost prohibits the observation of peoples'. This experimental study proceeds with the following point of view.

a) Occupants maintain higher cost-effectiveness of stable and rapid occupants' egress. 'Familiiarity' is still an important factor for occupants' decision of way of acting,as we have seen in many cases of fire. Therefore, in these series of experimental study human responses under quite normal situations were investigated. As mentioned above, the origin of psychological stress in normal situation is considered that it threatens the safety of egress in emergent situation too.

b) Visual image is taken account as afterwards dominative impulse changes into long term memory. The memory can be said to be a reference frame for identifying occupants' standinig point and choosing a direction in relation to the whole floor plan, and it is also supposed to be the very nature of 'familiarity. Therefore a simulator serving visual image can replace whole scale structure under confined applications.

\section{Flow of Study}

The experimental study took place according to the following procedure.

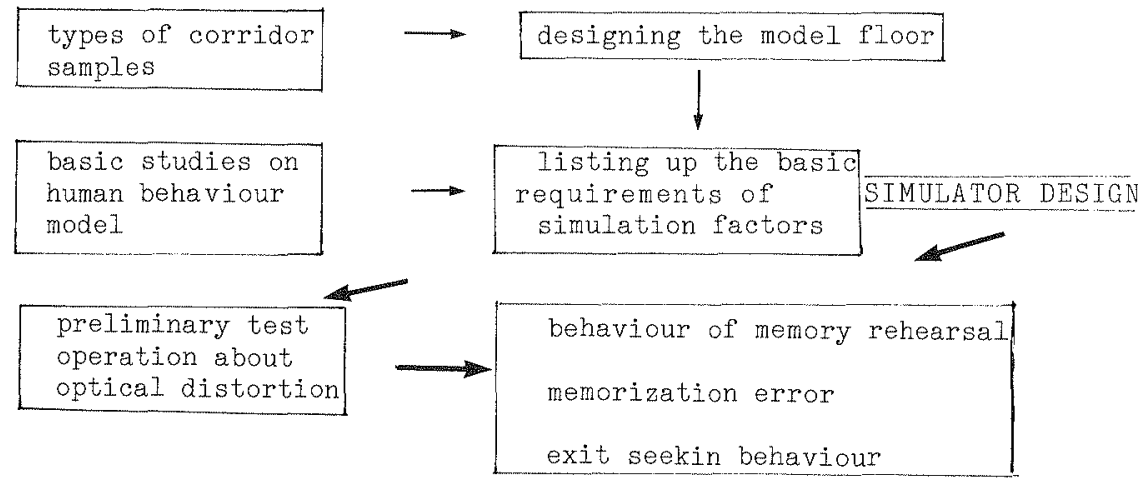


A) Development of the Visual Simulator

Simulator for visual experience of egress are composed of three parts : the floor model, the camera unit and the image projector. Figure I shows the concept.
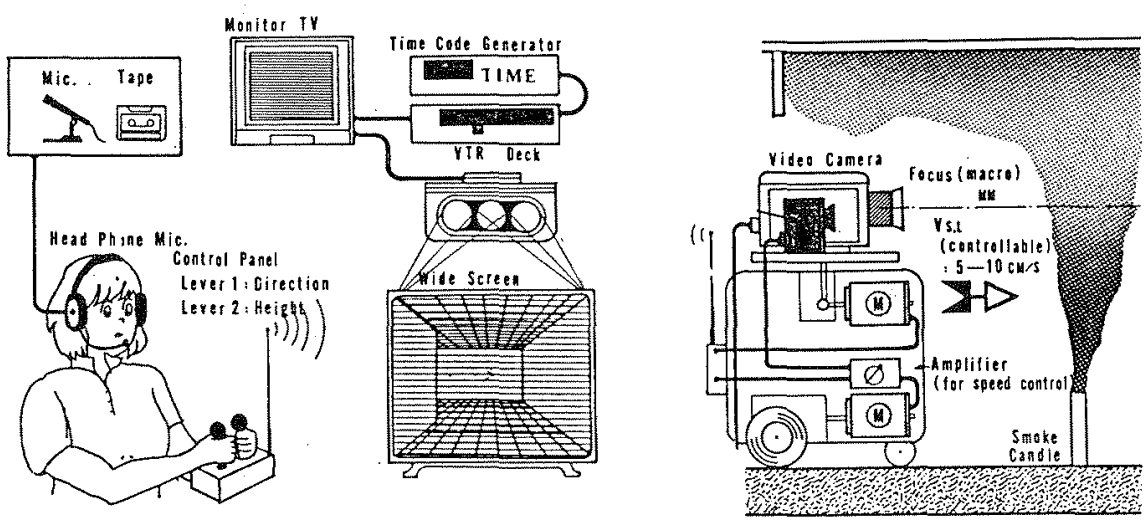

Fig. 1. Simulator concept

\section{Floor Mode1}

Floor model is an assembly of various corridors selected from building examples. Figure 2 shows the elements and the completion of models. All the walls are colored white with no distinguishing differences of finishing, thus people watching the image should be able to remember only the form and the types of the corridors. The scale of the model is basically 1/15, although the scale is adjustable by varying the moving speed of the camera unit.

\section{Camera Unit}

The camera unit consists of an engine, image emitters, a video camera, and a radio controlled switching systems. Moving speed is controIlable to about $8-10 \mathrm{~cm} / \mathrm{sec}$, that is $1 / 15-1 / 20$ scale of the real value of occupants' walking speed. Touch sensors were applied to maintain the stability of moving in changing directions by keeping constant distance from the guidance attached on the model floor. Figure 3 shows the completion of camera unit.

\section{Image Projector}

The image projector is composed of a 15-in. monitor, a 45-in. reflecting screen, and other communication systems. The participants sit in front of the screen, and indicate the walking or searching direction. The original plan was to let the participants operate the camera unit, but decrease the chance for operational errosss trained operator was used who drive camera unit following the orders of the participants. Figure 4 shows the projector part. 

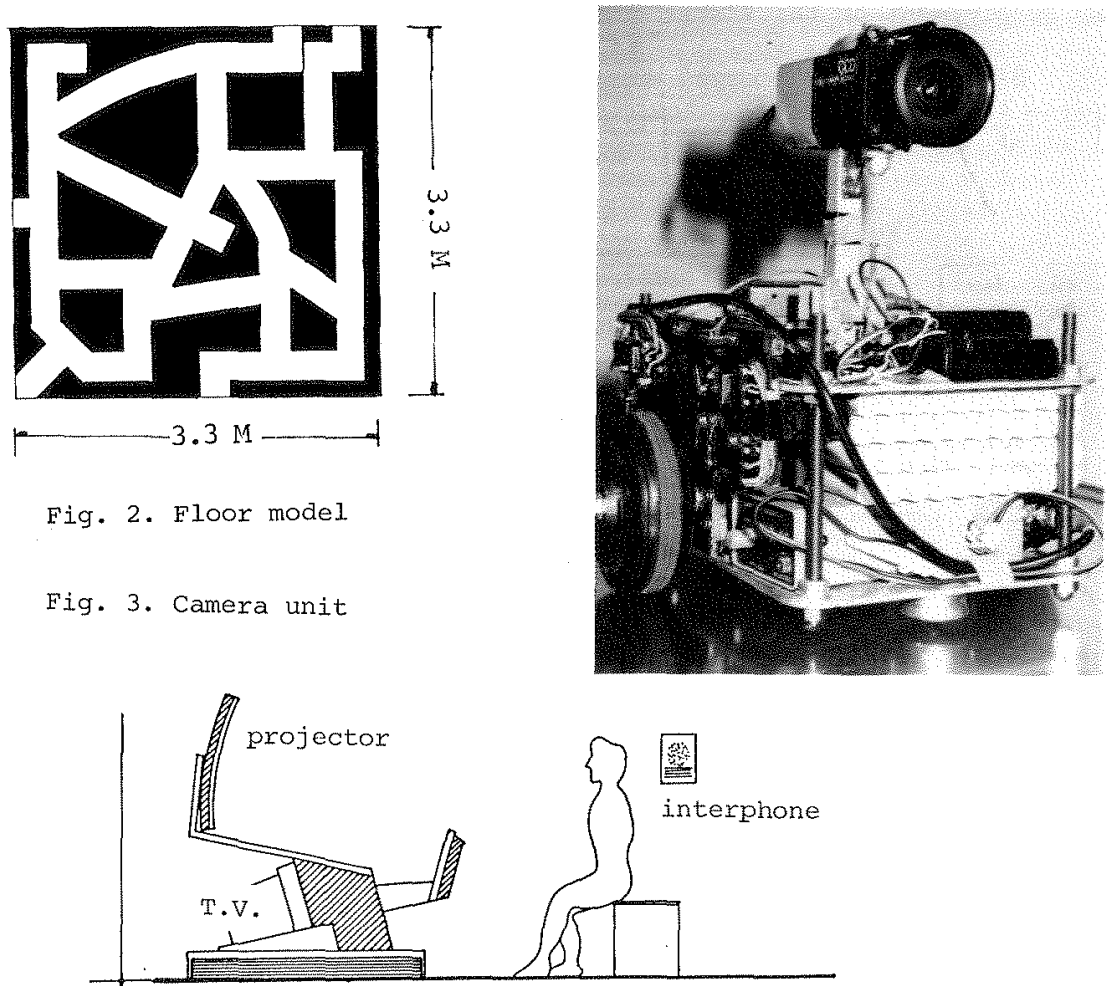

Fig. 4. Projector part

B) Preliminary Test Operation - Optical Distortion Check -

As the optical unit of wide angle generates a distortion of image on the outer part of the screen, the extent of distortion was checked prior to main experiments. After showing the continuous image, The operator paused the camera unit for a second on points A, B, C and let them guess the size of the angle. On point D, the operator rotated the camera to 180 . Then the rotating angle was also inquired. Figure 5 shows the surveying points, and Fig. 6 shows the comparison of the answered angle and the real.

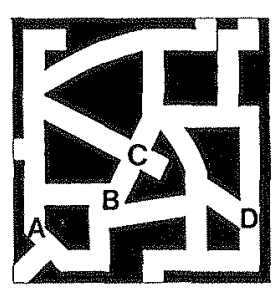

\begin{tabular}{|c|c|c|c|c|}
\hline angle & $\begin{array}{c}\text { A } \\
90^{\circ}\end{array}$ & $\begin{array}{c}\text { B } \\
120^{\circ}\end{array}$ & $\begin{array}{c}\text { C } \\
180^{\circ}\end{array}$ & $\begin{array}{c}\text { D } \\
60^{\circ}\end{array}$ \\
\hline the correct & $10 / 10$ & $10 / 10$ & $9 / 10$ & $8 / 10$ \\
\hline the answered & & & & \\
\hline
\end{tabular}

Fig. 5. Surveying points Table 1. The variation of the answered angle 
C) Experiment 1

This experiment aimed to observe the following items.

i) behaviour that changes according to motivation.

ii) reference of behaviour and the obviousness of memory.

iii) the characteristics of points where participants experienced confusion in direction.

iv) behaviour after being lost.

Experimental

We devided 25 volunteers into two groups ; A and B. The operator explained to group A that they should rehearse the route after experiencing it only one time, and $B$ recieved no information. Group $A$ and $B$ experienced the route of Fig. 6 and were then required to rehearse the course by oneself.

Result and Discussion

Figure 7 stands for the place and number of successors, and who failed in rehearsing at the point.

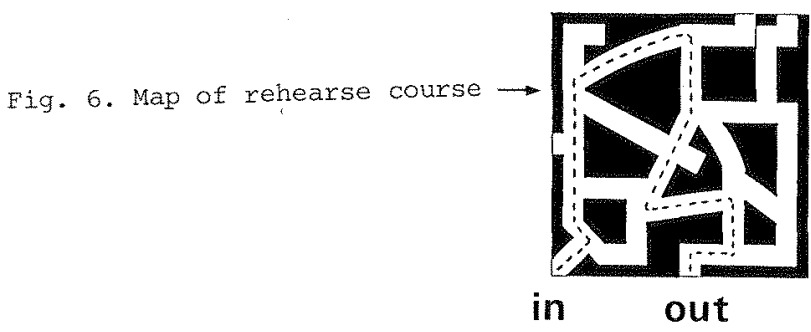

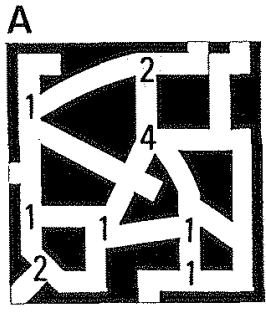

in.14 out.1

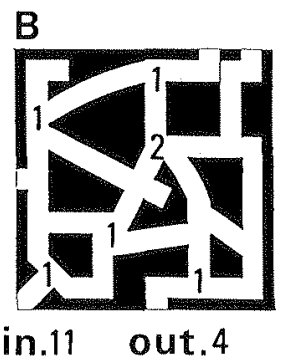

in.11 out. 4

Fig. 7. Result of rehearsal

Generally speaking, the memory of participants was comparatively consistent around the entrance. There, spending time on a junction of group A was comparatively short and supposed to have full of confidence. Group $B$ showed almost no changes in spending time for choosing the way, most of group A spend a longer time in choosing the way. But in most cases, they choose the correct route. The participant being lost showed the tendency to go around the same block. 
Motivation of experience influenced the strength of the participants memories and also their behaviour. For safety egress, it is required not only to give occupants direct information for emergent guidance, but also give them string incentive to help memorizing their experience in daily life under normal situations. Symmetrical design or junction with many auxillary corridors is not recommendable. It lowers the effectiveness of the occupants efforts to escape, and becomes a cause for metal pressure. Participants of group A showed confusion and a tendency to choose the way by impulse. Thinking time was very short but they repeated the same fallure thus showed an accelerated anxiety. In case of building plans with highly complex configuration, continuos information about the relation between way component direction and the building placement is necessary.

\section{D) Experiment 2}

One of the problems recently pointed out is the confusion of the occupant' recognition about his or her standing point, especially in the case where experience assemblies of way components are connected at random angles. It seems hard to say that the trouble in recognition of standing point is due to lack of memory or limit of perceptional ability of the angle size, though to find out the existance of psychological stress caused by random angles of way component was attempted. Figure 8 shows representative example of $120^{\circ}$ connected way components of 'New Japan Hotel,' produced a lot of fire victims.

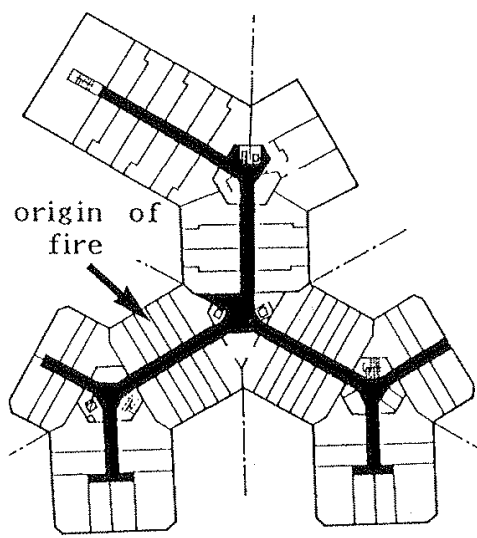

Fig. 8. Floor plan of hotel New Japan

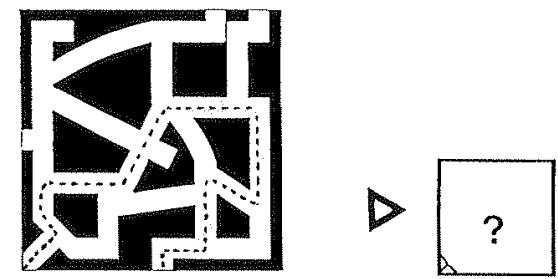

in out
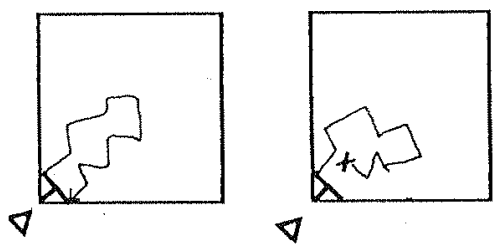

$\nabla$

Fig. 9. Examples of answered drawing 
Experimental

15 students participated in an experiment. After the experience of the course in Fig. 9, they were asked to describe the experienced course on $a$ paper without any grid or references except the mark stands for the starting point of 'S.P.'. On the guided course each participant experienced 9 corners, composed of 5 right angles, $3-120$ angles and $1-60$ angles. The image maps drawn showed following deviation :

Result and Discussion

As the result have shown, the images of random angles are changed into rectangular ones when recollected from memory. The problem of random angles as the cause for recognition error should be refered to in building design. Examples of image map drawn by each participant of experiment are shown in Fig. 9. The number of corners for each angle type on the indicated course and those angle types found in the image maps are compared here in Table 2.

Table 2. Result of experiment

\begin{tabular}{|c|c|c|}
\hline & the real & $\begin{array}{c}\text { found on } \\
\text { i mage map }\end{array}$ \\
\hline $90^{\circ}$ & $5(56 \%)$ & $5.1(70.8 \%)$ \\
$120^{\circ}$ & $3(33 \%)$ & $1.2(16.7 \%)$ \\
$60^{\circ}$ & $1(11 \%)$ & $0.9(12.5 \%)$ \\
total & 9 & 7.2 \\
\hline
\end{tabular}

E) Experiment 3

This experiment aimed to investigate the behaviour of seeking emergency exit, and the response of evacuees in case of the sudden interruption the way to the exit. 20 participants were given information about the floor plan and required to egress from exit 'B'. They were allowed to consult a guidance map for 30 seconds before starting from point ' $A$ '. When each participant approached point ' $C$ ', information was given that exit 'B' was not open due to sudden disaster. The operator requested the participants to do their best to escape through any exit on any exit on the floor as soon as possible. Under this situation, each participant's behaviour of seeking for emergency exits was observed and interpretated. Figure 10 shows the guide map.

Fig. 10. Guidance map

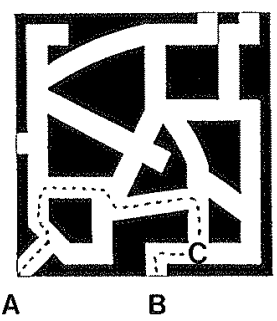


The indicated egress way was very simple, and 16 of the participants were successful in moving along the courase to exit.Four of the participants succeeded in reaching the exit though, but failed in choosing the idicated course. In this experiment, the following tendency of seeking egress behaviour was put under observation.

i) When the egress was closed,most of participants tried to return along the way she or he had entered.

ii) In comraratively long and straight corridors, each participant showed tendency of proceeding forward, and showed response of avoidanse against shorter ones.

iii) By accelerating the anxiety from. failure of egress, tendency of changing the proceeding direction or giving up before identifying the exit was observed often.

Viewed from these tendencies,continious information for egress way is quite important to stabilize the occupants and thus prevent their delay of evacuation time. And the way to help the occupants to choose the resonable egress way through the information with flexibility in case of exit trouble is also supposed to be taken into consideration for emergency evacuation safety.

Part II : Identification of Physiopsychological Impact in Case of Acute co Poisoning.

In general cases, the test method of toxity of gases generated during material combustion is estimated by the harmfulness of a biomaterial sample. The toxicity level is determined by the gas concentration and the measured time lag from exposure to the gas to the immobilization of the sample animal, meanwhile. If there exits physiopsychological impact, mobility supposed to have minor meaning as a parameter of an escaping ability, especially in case of building floor of highly complicated configuration. According to the many of the clinical literatures, some examples of physiopsychological impact of 60 gas have been denoted. In this experimental study, an attempt to find out the existance of physiopsychological impact of $\mathrm{CO}$ gas was made through the following procedure.

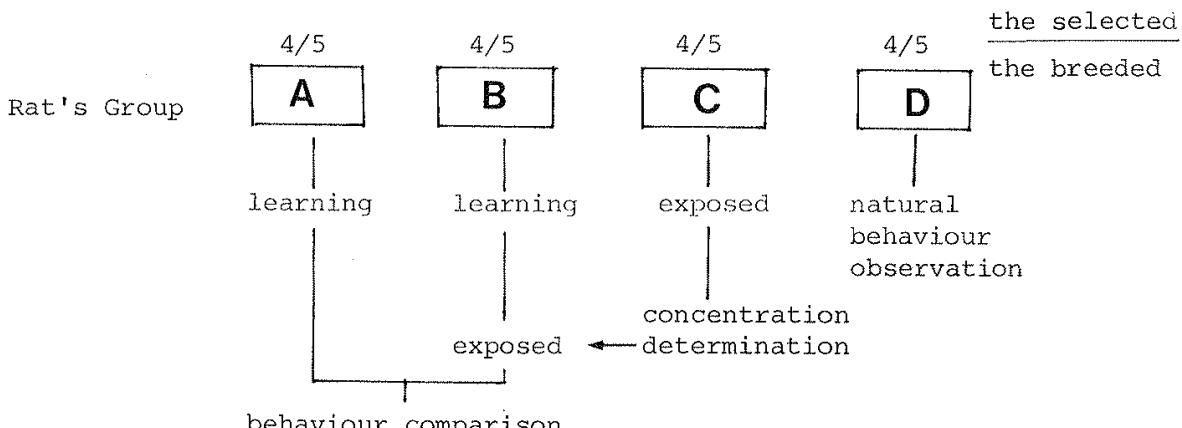

Fig. 11. Flow of experiment 


\section{A) Experimental}

Four groups of 20 rats were prepared for comparison analysis of CO poisoning. The procedure is shown in figure 11. Rats of group A underwent learning and was left as a contrast group without gas exposure. Group $B$ underwent learning and was exposed to the gas afterwards. Group $A$ having no prior experiance of gas exposure were then compared to group B to investigate the remaining trace of memory, to discuss $C O$ gas impact was discussed. As a control, group $C$ was used for setting appopriate exposure concentration. And group $A$ was put in natural environment, and the normal behaviour was observed.

Figure 12 shows the test apparatus that trains rats. Area $A$ is lightened $300 \mathrm{Lx}$, and are $B$ is of no light. The floor of B area is made of steel bars, and when rats touch the bars,around 0.6-1mAcurrent shocks the rat. Under natural situation, a rat put in area $A$ moves to $B$ area in a few minutes and settles there.

At first, rat was put in area $B$, and electric shocked. The shocked one escapes to area A though, return soon in accordance with the vanishiment of memory, the sense of risk against the area B. This apparatus is deviced to train the rat to induce the avoidance memory against the instinct, and to certify the vanishment of memory easily by simple observation.

Figure 13 shows the shape of the gas chamber. Rats are taken from the drawer of $A$. The chamber is made of a transparent acrylic pannel for easy observation from outside.

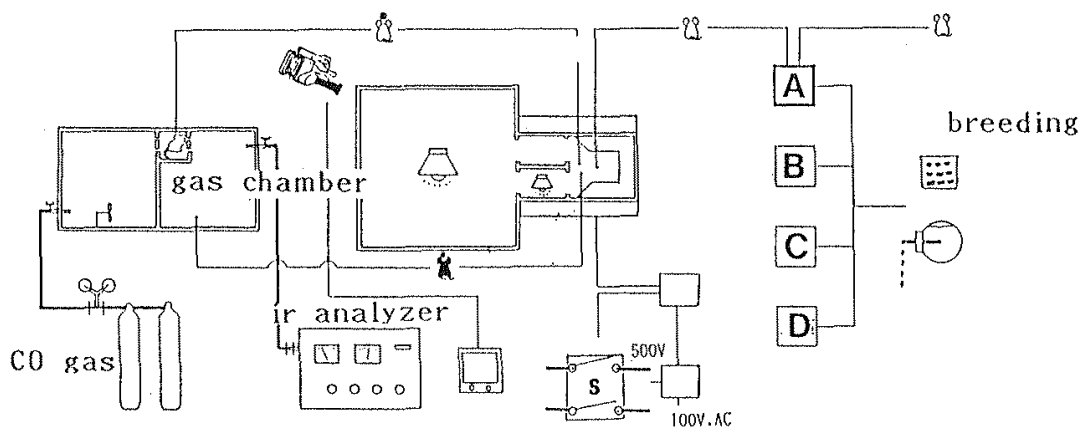

Fig. 12. Concept of experimental apparatus
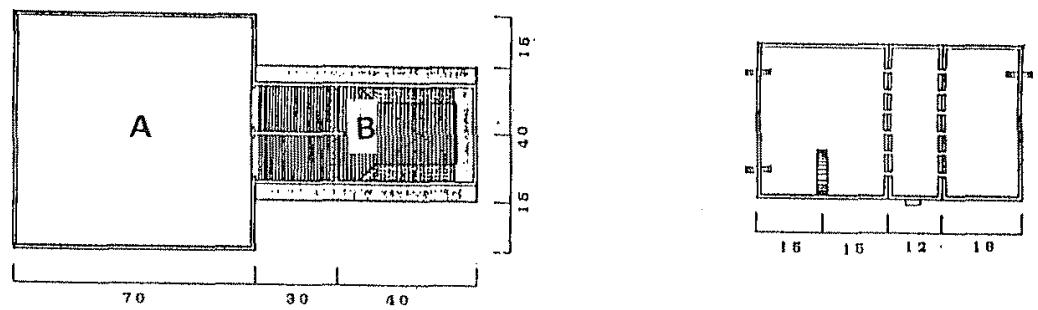

Fig. 13. Device for training and gas chamber 
In the learning process for rats of groups $A$ and $B$, obvious formation of memory was observed within 10-15 times trial. During the first $4-$ 5 times, the rats showed escape response by electric shock. But by increasing the time of trial, they became to move without the shock, this shows the formation of memory. Table 3 shows the comparison of behaviour seen in group A with acuteco poisoning and group B without exposure experience. As table 3 shows, vanishing of memory was supposed to be caused by co gas. In this experiment, the exposure time was 2 minute 40 seconds. At taht time, exposed rats shows very active movement, no other apparent visible symptoms of Co acute poisoning were observed. But as we found through the evaluation procedure of remained memory, the impact was supposed to be serious. In general, it is noted that a rat has more resistance than human. And co poisoning impact on mechanical memory is said to be weaker than that of the logical. These facts imply that human body would recieve same or more physiopsychological damage when being exposed to even lower concentration of CO gas.

\begin{tabular}{|l|l|}
\hline the exposed & \multicolumn{1}{|c|}{ not exposed } \\
\hline $\begin{array}{l}\text { The all } 4 \text { rats } \\
\text { moved from } A \text { to } \\
B \text { in } 2 \sim 34 \text { min } \\
\text { after being put } \\
\text { in } B .\end{array}$ & $\begin{array}{l}\text { Almost no move- } \\
\text { Stayed in }\end{array}$ \\
$\begin{array}{l}60 \text { min for under } \\
\text { observation, } \\
\text { making a group. }\end{array}$ \\
$\begin{array}{l}2 \sim 6 \text { min required } \\
\text { for recovery. } \\
\text { After recovery, } \\
\text { active movement } \\
\text { observed. }\end{array}$ & $\begin{array}{l}\text { Almost no } \\
\text { change in } \\
\text { behaviour. }\end{array}$ \\
\hline
\end{tabular}

Table 3. Injury observed

\section{REFERENCES}

1. de Boer, J. B., Cohu, M., de Graaff, A. B., Knudsen, B., Schreuder, D. A., : "Public Lighting," ed. J. B. de Boer, N. V. Philips' Gloeilampenfabrieken, Eindhoven, The Netherland, 1967

2. Kim, I. D. and Yun, D. R. : Carbon Monooxide Poisoning, Seoul National University, Seoul, Korea, 1970, p.290

3. Kishitani, K., Saito, F., Yusa, S. : Present State of Research in Japan on Toxicities of Gases During Fire, 6th Joint Meeting U.S.-Japan Panel on Fire Research and Safety, UJNR, Tokyo, 1982 